\title{
Modelo matemático para estimativa da temperatura média diária do ar no Estado de Goiás
}

\author{
Jorge Cesar dos Anjos Antonini(1), Euzebio Medrado Silva( ${ }^{(2)}$, \\ Luiz Fernando Coutinho de Oliveira ${ }^{(3)}$ e Edson Eyji Sano(2)
}

(1)Embrapa Transferência de Tecnologia, Parque Estação Biológica, Embrapa Sede, CEP 70770-901 Brasília, DF. E-mail: jorge.antonini@embrapa.br (2)Embrapa Cerrados, Caixa Postal 08223, CEP 73310-970 Planaltina, DF. E-mail: euzebio@cpac.embrapa.br, sano@cpac.embrapa.br ${ }^{(3)}$ Universidade Federal de Lavras, Departamento de Engenharia, Caixa Postal 3037, CEP $37200-000$ Lavras, MG. E-mail: Ifco@pq.cnpq.br

Resumo - O objetivo deste trabalho foi desenvolver um modelo matemático de estimativa da temperatura média diária do ar no Estado de Goiás, que considera simultaneamente as variações espacial e temporal. O modelo foi desenvolvido por meio de uma combinação linear da altitude, latitude, longitude e da série trigonométrica de Fourier incompleta usando os três primeiros coeficientes harmônicos. Os parâmetros do modelo foram ajustados aos dados de 21 estações meteorológicas, por meio de regressão linear múltipla. O coeficiente de correlação resultante do ajuste do modelo foi de 0,91 , e o índice de concordância de Willmott foi igual a 1 . $\mathrm{O}$ modelo foi testado com os dados de três estações de altitudes diferentes: elevada $(1.100 \mathrm{~m})$, média $(554 \mathrm{~m})$ e baixa $(431 \mathrm{~m})$. O desempenho foi considerado mediano para altitudes baixas e elevadas, e muito bom para altitudes médias.

Termos para indexação: modelagem climática, regressão linear múltipla, série de Fourier.

\section{Mathematical model for estimating daily average air temperature in Goiás, Brazil}

\begin{abstract}
The objective of this work was to develop a mathematical model to predict the daily average of air temperature in Goiás, Brazil. The model was developed through a linear combination of altitude, latitude, longitude, and the incomplete trigonometric Fourier series using the first three harmonic coefficients. The parameters of the model were adjusted with data from 21 weather stations, using multiple linear regression. The resulting correlation coefficient of the model was 0.91 , and the Willmott's index of agreement was close to 1 . The model was tested with data from three additional weather stations at different altitudes: high $(1,100 \mathrm{~m})$, medium $(554 \mathrm{~m})$, and low $(431 \mathrm{~m})$. The performance of the model was reasonable for both high and low altitude stations, and very good for the medium altitude station.
\end{abstract}

Index terms: climatic modeling, multiple linear regression, Fourier series.

\section{Introdução}

O conhecimento das variáveis meteorológicas é importante nos estudos relacionados ao desempenho das culturas agrícolas, e a temperatura do ar é a que mais interfere nos processos fisiológicos que ocorrem nas plantas. A temperatura influencia, principalmente, a velocidade das reações químicas e os processos internos de translocação de assimilados (Pereira et al., 2002; Benavides et al., 2007). Também, o conhecimento das temperaturas mínimas e máximas pode auxiliar no zoneamento agrícola de uma região (Galvani et al., 2000), na estimativa do ciclo das culturas e na definição da época de semeadura.
O monitoramento da temperatura do ar é feito diariamente nas estações meteorológicas convencionais ou automáticas. No entanto, quando a rede de estações meteorológicas é insuficiente para permitir a caracterização térmica da região, a temperatura do ar pode ser estimada por equação de regressão múltipla com base na altitude, latitude e longitude (Coelho et al., 1973; Pinto \& Alfonsi, 1974; Feitoza et al., 1979; Almeida \& Sá, 1984; Camargo \& Ghizzi, 1991; Luiz \& Silva, 1995; Sediyama et al., 1998; Cargnelutti Filho et al., 2006).

A estimativa da temperatura média mensal em função da altitude, longitude e latitude, com uso de modelos lineares, tem sido feita com aceitável grau de precisão 
(Medeiros et al., 2005; Stahl et al., 2006). Citam-se como exemplos os trabalhos de Lima \& Ribeiro (1998) no Estado do Piauí; Ferreira et al. (2006) nos estados de Minas Gerais e Pará; Pezzopane et al. (2004) no Espírito Santo; Oliveira Neto et al. (2002) na área situada entre 16 e $24^{\circ} \mathrm{S}$ e entre 48 e $60^{\circ} \mathrm{W}$; Cargnelutti Filho et al. (2008) no Rio Grande do Sul, para estimar as temperaturas média e máxima na escala decendial; Antonić et al. (2001) na República da Croácia, associada à técnica de redes neurais.

A temperatura média diária do ar varia continuamente durante o ano e apresenta uma nítida sazonalidade. Para o estudo de séries temporais, que apresentam variações periódicas ou sazonais, pode-se recorrer à análise harmônica por séries de Fourier, para o desenvolvimento de modelos de estimativa (Carvalho et al., 2005).

Os modelos propostos para a estimativa da temperatura do ar foram desenvolvidos com uso de variáveis espaciais ou temporais, separadamente. No entanto, para aplicação no zoneamento agrícola de risco climático (Assad et al., 2001), há necessidade de modelos matemáticos de simples utilização, que utilizem simultaneamente variáveis espaciais e temporais.

O objetivo deste trabalho foi desenvolver um modelo matemático de estimativa da temperatura média diária do ar no Estado de Goiás, que considera simultaneamente as variações espacial e temporal.

\section{Material e Métodos}

$\mathrm{O}$ estudo foi realizado no Estado de Goiás, entre $12^{\circ} 0^{\prime} 0^{\prime \prime} \mathrm{S}$ e $19^{\circ} 45^{\prime} 44^{\prime \prime} \mathrm{S}$ e $45^{\circ} 45^{\prime} 32^{\prime \prime} \mathrm{W}$ e $53^{\circ} 30^{\prime} 0^{\prime \prime} \mathrm{W}$. Foram utilizados dados de 24 estações meteorológicas (Tabela 1). Os dados do período 1985-2000 foram obtidos no Instituto Nacional de Meteorologia (Inmet), os de 2000-2007 foram obtidos no Sistema de Meteorologia e Hidrologia do Estado de Goiás (Simehgo), e os de 1983-2006 na estação meteorológica da Embrapa Arroz e Feijão.

A World Meteorological Organization preconiza que na análise de séries históricas de dados climáticos sejam utilizados dados de pelo menos 30 anos (Martin et al., 2008). As séries históricas de dados oficiais de temperatura do ar, disponíveis no Estado de Goiás, com densidade aceitável, não atingem esse número de anos de observações. Porém, resultados satisfatórios têm sido obtidos com séries históricas com o número de anos de observações abaixo do preconizado (Lima \& Ribeiro, 1998; Pezzopane et al., 2004; Gomes et al., 2005).

Para compor o modelo de estimativa da temperatura média diária do ar, em função do espaço e do tempo,

Tabela 1. Altitude, localização e período de observação das estações meteorológicas consideradas neste estudo, situadas no Estado de Goiás e no Distrito Federal.

\begin{tabular}{|c|c|c|c|c|c|}
\hline $\mathrm{N}^{\mathrm{o}}$ de ordem & Localidade & Altitude (m) & Latitude Sul $\left(^{\circ}\right)$ & Longitude Oeste $\left({ }^{\circ}\right)$ & Período (ano) \\
\hline 1 & Aragarças $^{(1)}$ & 311 & 15,900 & 52,230 & $1985-2000$ \\
\hline 2 & Brasília $^{(1)}$ & 1.161 & 15,789 & 47,926 & $1985-2000$ \\
\hline 3 & Britânia $^{(2)}$ & 284 & 15,429 & 51,220 & $2000-2007$ \\
\hline 4 & Caldas Novas ${ }^{(2)}$ & 706 & 17,726 & 48,616 & $2000-2007$ \\
\hline 5 & Catalão ${ }^{(1)}$ & 886 & 18,158 & 47,926 & $2000-2007$ \\
\hline 6 & Ceres $^{(2)}$ & 589 & 15,308 & 49,598 & $2000-2007$ \\
\hline 7 & Formosa $^{(1)}$ & 899 & 15,530 & 47,330 & $1985-2000$ \\
\hline 8 & Goianésia $^{(1)}$ & 665 & 15,220 & 48,990 & $1985-2000$ \\
\hline 9 & Goiânia $^{(1)}$ & 722 & 16,643 & 49,222 & $1985-2000$ \\
\hline 10 & Goiás ${ }^{(1)}$ & 512 & 15,939 & 50,141 & $1985-2000$ \\
\hline 11 & Goiatuba $^{(2)}$ & 774 & 18,013 & 49,357 & $2000-2007$ \\
\hline 12 & Ipameri $^{(1)}$ & 743 & 17,717 & 48,167 & $1985-2000$ \\
\hline 13 & Iporá $^{(2)}$ & 596 & 16,442 & 51,118 & $2000-2007$ \\
\hline 14 & Itaberai $^{(2)}$ & 701 & 16,020 & 49,810 & $2000-2007$ \\
\hline 15 & Itumbiara $^{(2)}$ & 455 & 18,420 & 49,218 & $2000-2007$ \\
\hline 16 & Jataí ${ }^{(1)}$ & 669 & 17,924 & 51,718 & $1985-2000$ \\
\hline 17 & Palmeiras de Goiás ${ }^{(2)}$ & 621 & 16,810 & 49,911 & $2000-2007$ \\
\hline 18 & Porangatu ${ }^{(2)}$ & 368 & 13,374 & 49,128 & $2000-2007$ \\
\hline 19 & Posse $^{(1)}$ & 824 & 14,089 & 46,366 & $1985-2000$ \\
\hline 20 & Santa Helena ${ }^{(2)}$ & 590 & 17,839 & 50,576 & $2000-2007$ \\
\hline 21 & Santo Antônio de Goiás ${ }^{(3)}$ & 829 & 16,500 & 49,282 & $1983-2006$ \\
\hline 22 & Anápolis $^{(2)}$ & 1.100 & 16,381 & 48,945 & $2004-2007$ \\
\hline 23 & Jandaia $^{(2)}$ & 554 & 17,240 & 50,203 & $2005-2007$ \\
\hline 24 & Minaçu $^{(2)}$ & 431 & 13,544 & 48,195 & $2005-2007$ \\
\hline
\end{tabular}

${ }^{(1)}$ Instituto Nacional de Meteorologia (Inmet). ${ }^{(2)}$ Sistema de Meteorologia e Hidrologia do Estado de Goiás (Simehgo). ${ }^{(3)}$ Embrapa Arroz e Feijão. 
utilizaram-se, como dados da variável dependente, os valores médios da temperatura média diária, para cada dia do ano $\left(\mathrm{To}_{\mathrm{ip}}\right)$ do período de observação das séries históricas consideradas. Como variáveis independentes, utilizaram-se altitude (Alt), latitude (Lat), longitude (Long) e a sequência dos dias do ano $\left(\mathrm{t}_{\mathrm{i}}\right)$. O modelo foi desenvolvido por meio de uma combinação linear da Alt, Lat e Long com a série trigonométrica de Fourier incompleta com os três primeiros harmônicos:

$$
\begin{aligned}
& \operatorname{Tm}_{\mathrm{i}}=\beta_{0}+\beta_{1} \text { Alt }+\beta_{2} \text { Lat }+\beta_{3} \text { Long }+\sum_{n=1}^{3}\left[a_{n} \cos \left(n w t_{i}\right)+\right. \\
& \left.b_{n} \operatorname{sen}\left(n w t_{i}\right)\right]
\end{aligned}
$$

em que: o $\mathrm{Tm}_{\mathrm{i}}$ é a temperatura média do ar do dia $\mathrm{i}$ $\left({ }^{\circ} \mathrm{C}\right)$; i é o i-ésimo dia do ano; $\beta_{0}$ é o coeficiente linear, e $\beta_{1}, \beta_{2}$ e $\beta_{3}$ são os coeficientes angulares das variáveis espaciais; Alt é a altitude do local (m); Lat é a latitude do local em valores positivos (graus decimais); Long é a longitude do local em valores positivos (graus decimais); $a_{n}$ e $b_{n}$ são os coeficientes dos termos da série trigonométrica de Fourier incompleta; $t_{i}$ é o dia do ano da observação i, que varia de 1 a 365 dias; $n$ é o número de harmônicos da série trigonométrica de Fourier; w é a frequência angular fundamental igual a $2 \pi / 365$. Com os coeficientes angulares $a_{n}$ e $b_{n}$ da série trigonométrica de Fourier, pode-se obter a amplitude de cada harmônica, igual a $\left(a_{n}^{2}+b_{n}^{2}\right)^{1 / 2}$, que representam as amplidudes anual, semestral e trimestral da temperatura média diária do ar.

Para demonstrar a aplicabilidade do modelo proposto, foram utilizados os valores diários de temperatura máxima e mínima do ar, de períodos não uniformizados, com o mínimo de oito anos de observações de séries históricas, das primeiras 21 estações meteorológicas listadas na Tabela 1.

Em cada estação meteorológica, a partir dos dados diários de temperatura máxima e mínima do ar, medidos em abrigo meteorológico, e com os dias do ano em uma sequência de 1 a 365 dias, foi calculada a temperatura média do ar do dia i no ano $\mathrm{j}\left(\mathrm{To}_{\mathrm{ij}}\right)$ de cada série histórica analisada. Com os valores de $\mathrm{To}_{\mathrm{ij}}$, foi calculada a temperatura média para cada dia do ano $\left(\mathrm{To}_{\mathrm{ip}}\right)$, referente ao período de observação da série histórica de dados, com o uso da equação:

$\mathrm{To}_{\mathrm{ip}}=\sum_{\mathrm{j}=1}^{\mathrm{Po}_{\mathrm{o}}}\left(\mathrm{To}_{\mathrm{ij}} / \mathrm{P}_{\mathrm{o}}\right)$

em que: j é o j-ésimo ano de observação; $T_{\mathrm{ij}}$ é a temperatura média diária referente ao i-ésimo dia do ano $\mathrm{j} ; \mathrm{P}_{\mathrm{o}}$ é o número de anos de observação da série histórica de dados de cada estação.
Os valores de $\mathrm{To}_{\text {ip }}\left({ }^{\circ} \mathrm{C}\right)$ totalizaram 7.665 dados $\mathrm{e}$, juntamente com os valores de latitude, longitude e altitude e os valores dos componentes dos três primeiros harmônicos da série trigonométrica de Fourier: $\cos (w t), \cos (2 w t), \cos (3 w t), \operatorname{sen}(w t), \operatorname{sen}(2 w t)$ e $\operatorname{sen}(3 w t)$ formaram uma matriz com 7.665 linhas e dez colunas, obtida com o programa Excel, para efetuar a determinação dos coeficientes do modelo pela análise de regressão linear múltipla. $O$ grau de ajuste do modelo foi avaliado pelo coeficiente de determinação, e a verificação da significância dos coeficientes pelo teste $t$ de Student, a 5\% de probabilidade de erro.

Para avaliar o grau de desvio do modelo, em relação à linha 1:1, foi utilizado o recurso da regressão linear simples, aplicado aos pares de valores estimados (equação 1) e observados.

A quantificação das diferenças entre os valores de temperatura média diária do ar, estimados pelo modelo (equação 1), e os valores observados nas séries históricas das estações meteorológicas estudadas foi feita com os seguintes índices estatísticos: média dos erros (MBE), variância dos erros $\left(\mathrm{s}^{2}\right)$, raiz do quadrado médio dos erros (RMSE) e média dos erros absolutos (MAE) definidos, respectivamente, pelas seguintes equações:

$$
\begin{aligned}
& \mathrm{MBE}=\sum_{\mathrm{i}=1}^{\mathrm{N}}\left(\mathrm{Tm}_{\mathrm{i}}-\mathrm{To}_{\mathrm{ip}}\right) / \mathrm{N} \\
& \mathrm{s}^{2}=\left[\sum_{\mathrm{i}=1}^{\mathrm{N}}\left(\mathrm{Tm}_{\mathrm{i}}-\mathrm{To}_{\mathrm{ip}}-\mathrm{MBE}\right)^{2}\right] /(\mathrm{N}-1) \\
& \mathrm{RMSE}=\sqrt{\sum_{\mathrm{i}=1}^{\mathrm{N}}\left(\mathrm{Tm}_{\mathrm{i}}-\mathrm{To}_{\mathrm{ip}}\right)^{2} / \mathrm{N}} \\
& \mathrm{MAE}=\sum_{\mathrm{i}=1}^{\mathrm{N}} \mid \mathrm{Tm}_{\mathrm{i}}-\mathrm{To}_{\mathrm{ip}} / \mathrm{N}
\end{aligned}
$$

Para o teste do modelo, foram feitas comparações entre a temperatura média diária observada e a estimada com o modelo ajustado (equação 1), tendo-se utilizado os dados das três estações meteorológicas não pertencentes ao grupo de estações usadas na determinação dos coeficientes lineares e angulares do modelo. Essas três estações foram selecionadas de modo que representassem as altitudes elevadas, médias e baixas. Para representar essas condições, foram selecionadas as estações de Anápolis, Jandaia e Minaçu (Tabela 1).

A qualidade preditiva do modelo foi avaliada quanto à precisão, exatidão e desempenho. A precisão foi quantificada pelo coeficiente de correlação de Pearson (r), calculado por meio da seguinte equação: 


$$
r=\sqrt{\sum_{i=1}^{N}\left(\mathrm{Tm}_{\mathrm{i}}-\overline{\mathrm{O}}\right)^{2} /\left[\sum_{\mathrm{i}=1}^{\mathrm{N}}\left(\mathrm{Tm}_{\mathrm{i}}-\overline{\mathrm{O}}\right)^{2}+\sum_{\mathrm{i}=1}^{\mathrm{N}}\left(\mathrm{To}_{\mathrm{ip}}-\mathrm{Tm}_{\mathrm{i}}\right)^{2}\right]}
$$

em que: $\mathrm{N}$ é o número total de observações; e $\overline{\mathrm{O}}$ é a média dos valores de temperatura média diária observados $\left({ }^{\circ} \mathrm{C}\right)$.

A exatidão foi quantificada pelo índice de concordância (d) de Willmott (Willmott, 1982), calculado por meio da equação:

$\mathrm{d}=1-\left[\sum_{\mathrm{i}=1}^{\mathrm{N}}\left(\mathrm{Tm}_{\mathrm{i}}-\overline{\mathrm{O}}\right)^{2} / \sum_{\mathrm{i}=1}^{\mathrm{N}}\left(\left|\mathrm{Tm}_{\mathrm{i}}-\overline{\mathrm{O}}\right|+\left|\mathrm{To}_{\mathrm{ip}}+\overline{\mathrm{O}}\right|\right)^{2}\right]$

O desempenho do modelo foi quantificado pelo índice de consistência $\mathrm{c}=\mathrm{r} \times \mathrm{d}$ (Camargo \& Camargo, 2000). Os indicadores de concordância e de desempenho foram interpretados de acordo com Santos (2007) e Camargo \& Sentelha (1997), respectivamente.

\section{Resultados e Discussão}

O ajuste do modelo desenvolvido (equação 1), feito por meio de análise de regressão linear múltipla, resultou em dez coeficientes significativos $\left(\beta_{0}=39,862522\right.$; $\beta_{1}=-0,007314 ; \beta_{2}=-0,185049 ; \beta_{3}=-0,144706 ;$ $\mathrm{a}_{1}=1,448522 ; \mathrm{a}_{2}=-1,182537 ; \mathrm{a}_{3}=0,223580$; $\left.b_{1}=-0,407352 ; b_{2}=0,119402 ; b_{3}=0,099942\right)$, com níveis de probabilidade próximos de zero, o que indica que os componentes são todos necessários para representar a variação espaço-temporal da temperatura média diária do ar $\left(\mathrm{Tm}_{\mathrm{i}}\right)$ no Estado de Goiás. Ao desenvolver equações para a estimativa da temperatura média mensal dos estados de Minas Gerais e Pará, Ferreira et al. (2006) consideraram apenas variáveis espaciais: altitude, latitude e longitude e, também, obtiveram todos os coeficientes dessas variáveis significativos.

Em um modelo linear, cada componente contribui para o resultado na proporção da magnitude dos valores de seus coeficientes. Por exemplo, no caso da componente altitude, o coeficiente $\beta_{1}$ indica que a $\mathrm{Tm}_{\mathrm{i}}$ decresce $0,73^{\circ} \mathrm{C}$ para cada $100 \mathrm{~m}$ de variação positiva em altitude. Este valor, apesar de ter sido derivado de uma regressão linear, é coerente, pois está entre o gradiente adiabático seco e o úmido da troposfera. Não difere muito do valor encontrado por Cargnelutti Filho et al. (2008) para a temperatura média decendial no Rio Grande do Sul, também com uso de regressão. No caso do coeficiente da latitude $\left(\beta_{2}\right)$, o aumento de $1^{\circ}$ correspondeu à diminuição aproximada de $0,19^{\circ} \mathrm{C}$ na temperatura do ar. Resultados semelhantes foram encontrados por Pezzopane et al. (2004), ao desenvolver modelos matemáticos para a estimativa das médias mensais das temperaturas mínimas, médias e máximas do ar no Espírito Santo. Em relação à longitude, observouse o decréscimo da temperatura de $0,14^{\circ} \mathrm{C}$ para cada aumento de $1^{\circ}$, comportamento este também observado por Lima \& Ribeiro (1998), no Piauí.

$\mathrm{O}$ coeficiente de determinação $\left(\mathrm{R}^{2}\right)$ reflete o quanto da variação de $\mathrm{Tm}_{\mathrm{i}}$ é explicada pelas variáveis que compõem o modelo. Nesse caso, o valor encontrado foi de 0,82 (Tabela 2). Assim, $18 \%$ da variabilidade de $\mathrm{Tm}_{\mathrm{i}}$ ocorreram em razão de outros fatores, que podem ser: deslocamento de massas de ar, nebulosidade, ventos e chuvas (Pereira et al., 2002). Este valor de $\mathrm{R}^{2}$ está dentro da faixa de 0,75 a 0,86 encontrada por Oliveira Neto et al. (2002) ao ajustar equações de regressão em função

Tabela 2. Medidas estatísticas da avaliação do grau de ajuste do modelo proposto para a estimativa da temperatura média diária no Estado de Goiás.

\begin{tabular}{|c|c|c|c|c|c|}
\hline \multirow[t]{2}{*}{ Descrição da medida estatística } & \multirow[t]{2}{*}{ Símbolo } & \multirow{2}{*}{$\begin{array}{l}\text { Ajuste do } \\
\text { modelo }\end{array}$} & \multicolumn{3}{|c|}{ Condição de altitude } \\
\hline & & & Elevada & Média & Baixa \\
\hline Média dos erros $\left({ }^{\circ} \mathrm{C}\right)$ & MBE & 0,00 & $-1,14$ & 0,31 & 0,59 \\
\hline Variância dos erros & $s^{2}$ & 0,85 & 0,95 & 0,96 & 0,84 \\
\hline Raiz do quadrado médio dos erros $\left({ }^{\circ} \mathrm{C}\right)$ & RMSE & 0,92 & 1,12 & 0,22 & 0,43 \\
\hline Média dos erros absolutos $\left({ }^{\circ} \mathrm{C}\right)$ & MAE & 0,71 & 1,27 & 0,78 & 0,82 \\
\hline Valores abaixo de MAE (\%) & - & 58,90 & 53,70 & 57,53 & 60,82 \\
\hline Valores abaixo de $1{ }^{\circ} \mathrm{C}(\%)$ & - & 74,59 & 38,08 & 72,88 & 67,67 \\
\hline Valores abaixo de $1,5^{\circ} \mathrm{C}(\%)$ & $=$ & 90,97 & 65,48 & 86,03 & 80,82 \\
\hline Média dos valores observados $\left({ }^{\circ} \mathrm{C}\right)$ & $\overline{\mathrm{O}}$ & 24,82 & 22,85 & 25,05 & 26,64 \\
\hline Média dos valores estimados $\left({ }^{\circ} \mathrm{C}\right)$ & $\overline{\mathrm{P}}$ & 24,83 & 21,70 & 25,36 & 27,23 \\
\hline Coeficiente de determinação & $\mathrm{R}^{2}$ & 0,82 & 0,62 & 0,75 & 0,60 \\
\hline Coeficiente de correlação & $\mathrm{r}$ & 0,91 & 0,79 & 0,87 & 0,77 \\
\hline Coeficiente de Willmott & $\mathrm{d}$ & 1,00 & 0,77 & 0,97 & 0,84 \\
\hline Índice de desempenho & $\mathrm{c}$ & 0,91 & 0,66 & 0,84 & 0,65 \\
\hline Número de observações & $\mathrm{N}$ & 7.665 & 365 & 365 & 365 \\
\hline
\end{tabular}


da Alt, Lat e Long, para estimar os valores médios mensais de temperatura média entre 16 e $24^{\circ} \mathrm{S}$ e 48 e $60^{\circ} \mathrm{W}$, no Brasil; e dentro da faixa de 0,75 a 0,93 encontrada por Cargnelutti Filho et al. (2008) ao ajustar equações de regressão com essas mesmas variáveis espaciais, para estimar os valores médios decendiais de temperatura média do Rio Grande do Sul.

Ao analisar as diferenças entre os valores de temperatura média diária do ar estimados (equação 1) e os observados nas séries históricas das estações meteorológicas, verificou-se que a magnitude do erro absoluto médio foi de apenas $0,71^{\circ} \mathrm{C}$ (Tabela 2). Esse valor abrange 58,9\% das 7.665 diferenças observadas. Dessas diferenças, $74,4 \%$ são menores do que $1^{\circ} \mathrm{C}$ e $90,7 \%$ são menores do que $1,5^{\circ} \mathrm{C}$.

O grau de correlação entre os valores estimados e observados dá uma ideia da dispersão das estimativas em relação à regressão linear dos dados. Essa medida, no entanto, não expressa totalmente o grau de confiabilidade do modelo, pois o valor do coeficiente de correlação não está necessariamente relacionado à magnitude do desvio entre o valor padrão (linha 1:1) e o valor estimado pelo modelo de regressão. Nessa comparação, quanto mais próximo de 1 for o coeficiente angular e mais próximo de 0 for o coeficiente linear, menor é o desvio em relação à linha $1: 1$, e maior é a concordância entre os valores observados e os estimados pelo modelo. No presente trabalho, o coeficiente angular foi de 0,8215 , o coeficiente linear de 4,4313 (Figura 1), e a variância dos erros, nessa regressão linear, foi de apenas 0,85 (Tabela 2), o que indica que a dispersão média dos dados foi menor do que $1^{\circ} \mathrm{C}$. A aplicação do modelo no ajuste aos dados espaciais e climáticos, dentro dos limites do Estado de Goiás, resultou em $\mathrm{d}=1$ e $\mathrm{r}=0,91$ (Tabela 2), o que reflete o alto grau de exatidão e precisão do modelo.

O modelo descreveu bem o curso anual de variação da temperatura média diária do ar das três estações meteorológicas de altitudes diferentes (Figura $2 \mathrm{~A}$, $\mathrm{B}$ e C). No entanto, notou-se tendência do modelo, nos primeiros seis meses do ano, em subestimar os valores de temperatura na estação de maior altitude, e em superestimar na de menor altitude. Observouse boa correlação (Figura 2 D, E e F) entre os valores observados e estimados de temperatura média diária do ar. A precisão, representada pelo coeficiente de correlação (r), foi de $0,79,0,87$ e 0,77 nas estações de elevada, média e baixa altitudes, respectivamente.
Essas correlações podem ser interpretadas como moderada a forte (Santos, 2007). Quanto à exatidão, representada pelo coeficiente de Willmott (d) na escala de 0 a 1 , os valores encontrados foram de $0,77,0,90 \mathrm{e}$ 0,84 , respectivamente, nas estações de elevada, média e baixa altitudes. Esses valores demonstram que o modelo foi capaz de estimar a temperatura média diária do ar com relativa exatidão.

O desempenho do modelo, representado pelo índice de consistência (c), variou com a altitude da estação meteorológica (Figura $2 \mathrm{D}, \mathrm{E}$ e F) e pode ser classificado como mediano $(0,61$ a 0,65$)$, para as estações de altitudes baixa e elevada, e muito bom $(0,76$ a 0,85$)$ para a estação de altitude média. O melhor desempenho do modelo na estação de altitude média pode estar relacionado à maior densidade de estações meteorológicas dessa altitude usadas na fase de ajuste do modelo. Foram utilizados dados de apenas uma estação de altitude elevada, o que limita a capacidade de o modelo estimar valores mais próximos dessa realidade.



Figura 1. Diagrama de dispersão dos 7.665 valores de temperatura média diária do ar, observados $\left(\mathrm{To}_{\mathrm{i}}\right)$ e estimados $\left(\mathrm{Tm}_{\mathrm{i}}\right)$ pelo modelo proposto, e os correspondentes índices estatísticos de avaliação do ajuste (d, coeficiente de Willmott; r, coeficiente de correlação de Pearson; c, índice de desempenho). 

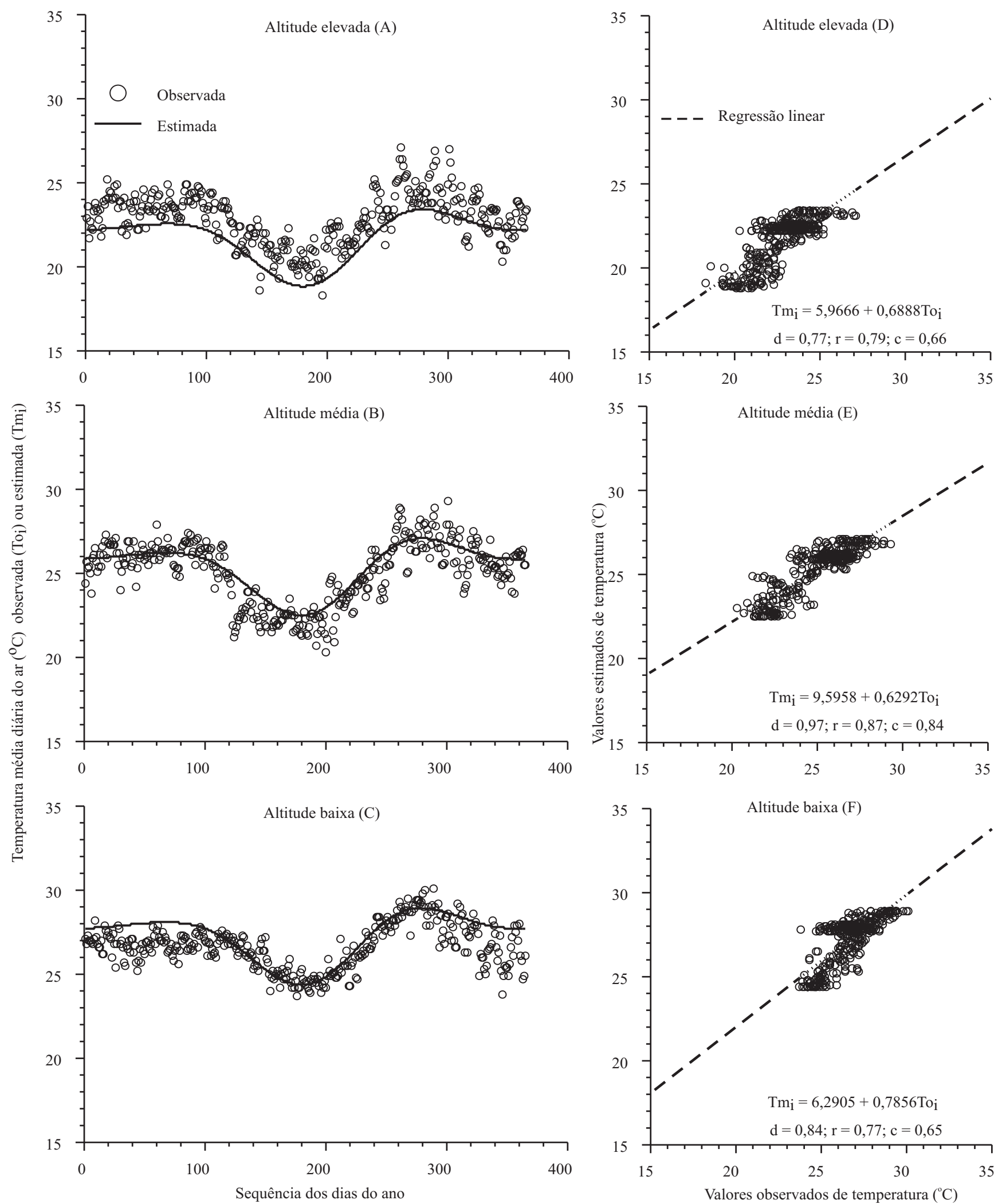

Figura 2. Variação anual da temperatura média diária do ar $(\mathrm{A}, \mathrm{B}, \mathrm{C})$ e os respectivos diagramas de dispersão (D, E, F) dos valores observados $\left(\mathrm{To}_{\mathrm{i}}\right)$ e estimados $\left(\mathrm{Tm}_{\mathrm{i}}\right)$, nas condições de altitude elevada, média e baixa, e os correspondentes índices estatísticos de validação do modelo (d, coeficiente de Willmott; r, coeficiente de correlação de Pearson; c, índice de desempenho). 


\section{Conclusão}

O modelo desenvolvido prevê adequadamente a variação da temperatura média diária no Estado de Goiás, com desempenho mediano para altitudes baixas e elevadas, e desempenho muito bom para altitudes médias.

\section{Agradecimentos}

Ao Gerente Geral da Embrapa Transferência de Tecnologia, José Roberto Rodrigues Peres, pela viabilização das condições necessárias à realização deste trabalho; à Embrapa Cerrados, pelo apoio logístico; ao Sistema de Meteorologia e Hidrologia do Estado de Goiás, pela disponibilização de dados; ao pesquisador da Embrapa Cerrados, Fernando Antônio Macena da Silva, pela orientação no tratamento de dados; ao geógrafo da Embrapa Cerrados, Heleno da Silva Bezerra, pelo apoio no laboratório de biofísica ambiental.

\section{Referências}

ALMEIDA, H.A.; SÁ, D.F. Estimativa das temperaturas médias, máximas e mínimas mensais e anuais do Sudoeste da Bahia. Revista Theobroma, v.14, p.135-142, 1984.

ANTONIĆ, O.; KRIZAN, J.; MARKI, A.; BUCOVEC, D. Spatiotemporal interpolation of climatic variables over large region of complex terrain using neural networks. Ecological Modelling, v.138, p.255-263, 2001.

ASSAD, E.D.; EVANGELISTA, B.A.; SILVA, F.A.M. da; CUNHA, S.A.R. da; ALVES, E.R.; LOPES, T.S. de S.; PINTO, H.S.; ZULLO JUNIOR, J. Zoneamento agroclimático para a cultura do café (Coffea arabica L.) no Estado de Goiás e Sudoeste do Estado da Bahia. Revista Brasileira de Agrometeorologia, v.9, p.510-518, 2001.

BENAVIDES, R.; MONTES, F.; RUBIO, A.; OSORO, K. Geostatistical modelling of air temperature in a mountainous region of Northern Spain. Agricultural and Forest Meteorology, v.146, p.173-188, 2007.

CAMARGO, A.P. de; CAMARGO, M.B.P. de. Uma revisão analítica da evapotranspiração potencial. Bragantia, v.59, p.125-137, 2000.

CAMARGO, A.P. de; GHIZZI, S.M. Estimativa de temperaturas médias mensais com base em cartas de temperatura potencial normal ao nível do mar para a região Sudeste do Brasil. Campinas: IAC, 1991. 17p. (IAC. Boletim Técnico, 141).

CAMARGO,A.P. de; SENTELHAS, P.C. Avaliação de desempenho de diferentes métodos de estimativa da evapotranspiração potencial no Estado de São Paulo, Brasil. Revista Brasileira de Agrometeorologia, v.5, p.89-97, 1997.

CARGNELUTTI FILHO, A.; MALUF, J.R.T.; MATZENAUER, R. Coordenadas geográficas na estimativa das temperaturas máxima e média decendiais do ar no Estado do Rio Grande do Sul. Ciência Rural, v.38, p.2448-2456, 2008.

CARGNELUTTI FILHO, A.; MALUF, J.R.T.; MATZENAUER, R.; STOLZ, A.P. Altitude e coordenadas geográficas na estimativa da temperatura mínima média decendial do ar no Estado do Rio Grande do Sul. Pesquisa Agropecuária Brasileira, v.41, p.893-901, 2006.

CARVALHO, L.G. de; SEDIYAMA, G.C.; CECON, P.R.; ALVES, H.M.R. Aplicação da análise harmônica por séries de Fourier para a previsão de produtividade da cultura do café no Estado de Minas Gerais. Engenharia Agrícola, v.25, p.732-741, 2005.

COELHO, D.T.; SEDIYAMA, G.C.; VIEIRA, M. Estimativa das temperaturas médias mensais e anual no Estado de Minas Gerais. Revista Ceres, v.20, p.455-459, 1973.

FEITOZA, L.R.; SCÁRDUA, J.A.; SEDIYAMA, G.C.; OLIVEIRA, L.M.; VALLE, S.S. Estimativa das temperaturas médias mensais e anual do Estado do Espírito Santo. Revista Centro de Ciências Rurais, v.9, p.279-291, 1979.

FERREIRA, W.P.M.; VALADARES, G.S.; HOTT, M.C. Estimativa da temperatura média mensal do ar para o Estado de Minas Gerais e do Pará, utilizando-se modelos originais de elevação. Engenharia na Agricultura, v.14, p.293-303, 2006.

GALVANI, E.; KLOSOWSKI, E.S.; ESCOBEDO, J.F.; CUNHA, A.R. da. Modelo de estimativa de temperatura mínima do ar para a região de Maringá - PR. Revista Brasileira de Agrometeorologia, v.8, p.105-110, 2000.

GOMES, A.A.N.; ANDRADE JÚNIOR, A.S. de; MEDEIROS, R.M. de. Evapotranspiração de referência mensal para o Estado do Piauí. Revista Brasileira de Engenharia Agrícola e Ambiental, v.9, p.560-564, 2005.

LIMA, M.G. de; RIBEIRO, V.Q. Equações de estimativa da temperatura do ar para o Estado do Piauí. Revista Brasileira de Agrometeorologia, v.6, p.221-227, 1998.

LUIZ, A.J.B.; SILVA, F.A.M. da. Temperaturas decendiais máxima, mínima e média, como função da latitude e altitude, em Goiás. In: CONGRESSO BRASILEIRO DE AGROMETEOROLOGIA, 9., 1995, Campina Grande. Anais. Campina Grande: Sociedade Brasileira de Agrometeorologia, 1995. p.234-236.

MARTIN, T.N.; DOURADO NETO, D.; STORCK, L.; BURAUEL, P.; SANTOS, E.A. Regiões homogêneas e tamanho de amostra para atributos do clima no Estado de São Paulo, Brasil. Ciência Rural, v.38, p.690-697, 2008.

MEDEIROS, S. de S.; CECÍLIO, R.A.; MELO JÚNIOR, J.C.F. de; SILVA JÚNIOR, J.L.C. da. Estimativa e espacialização das temperaturas do ar mínimas, médias e máximas na região Nordeste do Brasil. Revista Brasileira de Engenharia Agrícola e Ambiental, v.9, p.247-255, 2005.

OLIVEIRA NETO, S.N. de; REIS, G.G. dos; REIS, M. das G.F.; LEITE, H.G.; COSTA, J.M.N. da. Estimativa de temperaturas mínima, média e máxima do território brasileiro situado entre 16 e $24^{\circ}$ latitude sul e 48 e $60^{\circ}$ longitude oeste. Engenharia na Agricultura, v.10, p.8-17, 2002.

PEREIRA, A.R.; ANGELOCCI, L.R.; SENTELHAS, P.C. Agrometeorologia: fundamentos e aplicações práticas. Guaíba: Agropecuária, 2002. 478p. 
PEZZOPANE，J.E.M.; SANTOS，E.A.; ELEUTÉRIO，M.M.; REIS, E.F. dos; SANTOS, A.R. dos. Espacialização da temperatura do ar no Estado do Espírito Santo. Revista Brasileira de Agrometeorologia, v.12, p.151-158, 2004.

PINTO, H.S.; ALFONSI, R.R. Estimativa das temperaturas médias, máximas e mínimas mensais no Estado do Paraná, em função da altitude e latitude. Caderno de Ciências da Terra, v.52, p.1-28, 1974.

SANTOS, C. Estatística descritiva: manual de auto-aprendizagem. Lisboa: Silabo, 2007. 264p.

SEDIYAMA, G.C.; MELO JÚNIOR, J.C.F. Modelos para estimativa das temperaturas normais mensais médias, máximas, mínimas e anual no Estado de Minas Gerais. Engenharia na Agricultura, v.6, p.57-61, 1998.

STAHL, K.; MOORE, R.D.; FLOYER, J.A.; ASPLIN, M.G.; McKENDRY, I.G. Comparison of approaches for spatial interpolation of daily air temperature in a large region with complex topography and highly variable station density. Agricultural and Forest Meteorology, v.139, p.224-236, 2006.

WILLMOTT, C.J. Some comments on the evaluation of model performance. Bulletin of the American Meteorological Society, v.63, p.1309-1313, 1982.

Recebido em 26 de novembro de 2008 e aprovado em 31 de março de 2009 